Article

\title{
Some Approaches to the Calculation of Conservation Laws for a Telegraph System and Their Comparisons
}

\author{
Eerdun Buhe ${ }^{1,2,3, *(\mathbb{D})}$, G.W. Bluman ${ }^{4}$, Chen Alatancang ${ }^{1,2}$ and Hu Yulan ${ }^{3}$ \\ 1 School of Mathematical Sciences, Inner Mongolia University, Hohhot 010021, China; alatanca@imu.edu.cn \\ 2 Department of Mathematics, Hohhot University for Nationalities, Hohhot 010051, China \\ 3 College of Sciences, Inner Mongolia University for Technology, Hohhot 010051, China; yjshuyulan@163.com \\ 4 Department of Mathematics, University of British Columbia, Vancouver V6T 1Z2, Canada; \\ bluman@math.ubc.ca \\ * Correspondence: eerdunbuhe@hotmail.com; Tel.:+86-471-4933-405
}

Received: 8 April 2018; Accepted: 17 May 2018; Published: 24 May 2018

\begin{abstract}
This paper applies the direct construction method, symmetry /adjoint symmetry pair method (SA method), symmetry action on a known conservation law method, Ibragimov's conservation theorem (which always yields the same results as the SA method) and a recursion formula to calculate several conservation laws for nonlinear telegraph systems. In addition, a comparison is made between these methods for conservation laws admitted by nonlinear telegraph systems.
\end{abstract}

Keywords: conservation laws; nonlinear telegraph system; symmetries; adjoint symmetries; symmetry action; multipliers

\section{Introduction}

Conservation laws are essential in many fields of applications. In particular, they are important in studying the properties of solutions, integrability and in the development of numerical solutions for partial differential equations (PDEs). From the famous Noether's theorem for variational problems [1-5] introduced in Noether's celebrated 1918 paper, research on orientation has been transferred to the derivation and application of conservation laws for PDEs. Several systematic approaches have been established for the calculation of conservation laws, such as the symmetry/adjoint symmetry pair method (SA method) [5-7], the direct construction method (multiplier approach, variational derivatives approach) [3-5,7-9], symmetry action on a known conservation law method [5,10], Ibragimov's conservation theorem [11] (equivalent to the SA method [12]) and Cheviakov's recursion formul [13]. Wolf, Hereman, Temuerchaolu and Cheviakov [14-17] have presented some effective computer programmes to calculate conservation laws for PDEs.

In the SA method, every conservation law results from a bilinear skew-symmetric identity and includes the use of any pair consisting of a local symmetry and an adjoint symmetry of a given PDE system. The symmetry action on a known conservation law method $[5,10]$ directly seeks new conservation laws for any PDE system from an admitted symmetry action on a known conservation law. In Ibragimov's conservation theorem [11], a general formula on conservation laws for arbitrary PDEs is stated by combining the Lie symmetry generators and adjoint equations with formal Lagrangians.

A system of $m$-th-order PDEs in $N$-dependent variables $u=\left(u^{1}, u^{2}, \ldots, u^{N}\right)$ and $n$-independent variables $x=\left(x_{1}, x_{2}, \ldots, x_{n}\right)$ is given as:

$$
G_{\alpha}[u]=G_{\alpha}\left(x, u, \partial_{x} u, \ldots, \partial_{x}^{m} u\right)=0, \alpha=1, \ldots, M
$$


where $\partial_{x} u, \partial_{x}^{2} u$, etc., are all given order derivatives of $u^{\sigma}$ with respect to $x$ up to $m$. We suppose $U=\left(U^{1}, U^{2}, \ldots, U^{N}\right)$ are arbitrary functions of $x$, and $U_{i}^{\sigma}=\partial U^{\sigma} / \partial x_{i}, U_{i j}^{\sigma}=\partial^{2} U^{\sigma} / \partial x_{i} \partial x_{j}$, etc. Corresponding total derivatives are written by:

$$
D_{i}=\frac{\partial}{\partial x_{i}}+U_{i}^{\sigma} \frac{\partial}{\partial U^{\sigma}}+U_{i j}^{\sigma} \frac{\partial}{\partial U_{j}^{\sigma}}+\cdots+U_{i_{1} i_{2} \cdots i_{l}}^{\sigma} \frac{\partial}{\partial U_{i_{1} i_{2} \cdots i_{l}}^{\sigma}}+\cdots
$$

We let $F[U]$ represent a function depending on $x, U$ and derivatives of $U$ with respect to $x$.

Definition 1. If a one-parameter ( $\varepsilon$ ) Lie group of point transformations:

$$
\begin{aligned}
& x^{*}=x+\varepsilon \xi(x, u)+O\left(\varepsilon^{2}\right), \\
& u^{*}=u+\varepsilon \eta(x, u)+O\left(\varepsilon^{2}\right)
\end{aligned}
$$

leaves the PDE system (1) invariant, then (3) is named a point symmetry of PDE System (1), and $\xi=\left\{\xi^{i}(x, u)\right\}_{i=1}^{n}, \eta=\left\{\eta^{\sigma}(x, u)\right\}_{\sigma=1}^{N}$ are called the infinitesimals of the point symmetry (3).

Let:

$$
X=\xi^{i}(x, u) \frac{\partial}{\partial x_{i}}+\eta^{\sigma}(x, u) \frac{\partial}{\partial u^{\sigma}}
$$

be the infinitesimal generator of (3). The $k$-th extension of the infinitesimal generator (4) is:

$$
X^{(k)}=X+\eta_{i}^{(1) \sigma} \frac{\partial}{\partial u_{i}^{\sigma}}+\ldots+\eta_{i_{1} i_{2} \ldots i_{k}}^{(k) \sigma} \frac{\partial}{\partial u_{i_{1} i_{2} \ldots i_{k}}^{\sigma}}, k \geqslant 1,
$$

where:

$$
\begin{aligned}
& \eta_{i}^{(1) \sigma}=D_{i} \eta^{\sigma}-\left(D_{i} \xi^{j}\right) u_{j}^{\sigma} \\
& \eta_{i_{1} i_{2} \ldots i_{k}}^{(k) \sigma}=D_{i_{k}} \eta_{i_{1} i_{2} \ldots i_{k-1}}^{(k-1) \sigma}-\left(D_{i_{k}} \xi^{j}\right) u_{i_{1} i_{2} \ldots i_{k-1} j}^{\sigma} .
\end{aligned}
$$

Here, $\sigma=1,2, \ldots, N, i, i_{j}=1,2, \ldots, n$ for $j=1,2, \ldots, k$ with $k=2,3, \ldots$. , and $D_{i}$ is the total differentiation operator (2).

The generator:

$$
\hat{X}=\hat{\eta}^{\sigma} \frac{\partial}{\partial u^{\sigma}}=\left[\eta^{\sigma}(x, u)-u_{i}^{\sigma} \xi^{i}(x, u)\right] \frac{\partial}{\partial u^{\sigma}}
$$

is named the characteristic (evolutionary) form of (4).

Definition 2. A local conservation law of PDE System (1) is a divergence expression:

$$
D_{i} \Phi^{i}[u]=0,
$$

yielding for all solutions $u(x)$ of (1), and $\Phi^{i}[u]$ are called the conserved densities (fluxes).

The layout of the rest of this paper is as follows: In Section 2, the direct construction method and the recursion formula are discussed. In Sections 3 and 4, the SA method and symmetry action on a known conservation law method for obtaining conservation laws for PDEs are reviewed, respectively. In Section 5, these approaches are applied to seek conservation laws for a nonlinear telegraph system, and a comparison is made between the SA method, Ibragimov's conservation theorem and the symmetry action on a known conservation law method for conservation laws admitted by such a nonlinear telegraph equation. Finally, conclusions are summarized in Section 6. 


\section{Direct Construction Method}

In general, for a given PDE system (1) expressed in a standard Cauchy-Kovalevskaya form, all non-trivial conservation laws arise from linear combinations of the PDE system (1) with multipliers that hold non-trivial divergence expressions $[4,5,9,18]$. In this paper, we suppose that (1) is expressed in a standard Cauchy-Kovalevskaya form.

Definition 3. A set of multipliers (factors, characteristics) $\left\{\Lambda^{\alpha}[U]\right\}$ yields a divergence expression for the PDE system (1) if the identity:

$$
\Lambda^{\alpha}[U] G_{\alpha}[U]=D_{i} \Phi^{i}[U]
$$

holds for arbitrary functions $U(x)$.

For all solutions $U^{\sigma}=u^{\sigma}(x)$ of given System (1), if $\left\{\Lambda^{\alpha}[U]\right\}$ is non-singular, one has a local conservation law:

$$
D_{i} \Phi^{i}[u]=0
$$

of System (1).

There are many algorithms for determining the conservation laws of a given PDE system. The direct construction method especially, introduced in [3-5,7-9], yields the formulas and multipliers for the corresponding conservation laws when no variational principle exists. Specifically, $\left\{\Lambda^{\alpha}[U]\right\}$ holds a set of multipliers for a conservation law of System (1) if and only if each Euler operator (variational derivative):

$$
E_{U^{\sigma}}=\frac{\partial}{\partial U^{\sigma}}+\sum_{s \geq 1}^{\infty}(-1)^{s} D_{i_{1}} \ldots D_{i_{s}} \frac{\partial}{\partial U_{i_{1} \ldots i_{s}}^{\sigma}}
$$

annihilates the left-hand side of (9), i.e.,

$$
E_{U^{\sigma}}\left(\Lambda^{\alpha}[U] G_{\alpha}[U]\right) \equiv 0, \sigma=1, \ldots, N,
$$

for arbitrary $U^{\sigma}, U_{i}^{\sigma}, U_{i j}^{\sigma}, \ldots$, etc.

Condition (12) can be separated in regards to $G_{\alpha}[U]$ and its differential consequences to hold a set of over-determined linear homogeneous PDEs named the determining system (adjoint invariance conditions) for multipliers $\Lambda^{\alpha}[U]$. If (1) is self-adjoint system, i.e., PDE System (1) has a Lagrangian function, then its multipliers are generators of its admitted continuous (point, contact, higher order) symmetries in characteristic form subject to additional conditions. For any multiplier $\left\{\Lambda^{\alpha}[U]\right\}$ of adjoint invariance conditions (12), one can directly calculate the corresponding fluxes $\Phi^{j}[u]$ under an integral formula (see [5,7-9]). Zhang [19] has studied successfully the existence of multipliers for conservation law of PDEs by applying the property of nonlinear self-adjointness with differential substitution.

Unlike the other methods reviewed in this paper, the direct calculation method yields all local conservation laws and any order multipliers for PDE systems written in Cauchy-Kovalevskaya form.

Recently, a recursion formula [13] for the construction of local conservation laws of PDEs was presented by Cheviakov and Naz, which produces a divergence expression including an arbitrary function of all independent variables, summarized by the following lemma.

Lemma 1. Suppose that PDE System (1) admits a non-trivial local conservation law (8). Then, an arbitrary differentiable function $f=f(x)$, following the formal divergence expression, vanishes on any given solution $u(x)$ of $(1)$ :

$$
D_{i} \Xi^{i} \equiv D_{i}\left(f \Phi^{i}[u]-\int \frac{\partial f}{\partial x_{i}} \Phi^{i}[u] d x_{i}\right) .
$$




\section{Symmetry/Adjoint Symmetry Pair Method}

The symmetry/adjoint symmetry pair method [5-8,20] (SA method) contains the following steps: (a) linearize the given PDE system using Fréchet derivatives; (b) find the adjoint system of the linearized system by applying adjoint Fréchet derivatives; (c) find solutions of the linearized system; (d) find adjoint symmetries of the adjoint system; (e) for any pair, consisting of a adjoint symmetry and a local symmetry, seek a conservation law directly through the given conservation identity.

The linearizing operator (Fréchet derivative) associated with the PDE system (1) is given by:

$$
L_{\rho}^{\sigma}[U] V^{\rho}=\left[\frac{\partial G_{\sigma}[U]}{\partial U^{\rho}}+\frac{\partial G_{\sigma}[U]}{\partial U_{i}^{\rho}} D_{i}+\cdots+\frac{\partial G_{\sigma}[U]}{\partial U_{i_{1} \cdots i_{k}}^{\rho}} D_{i_{1}} \cdots D_{i_{k}}\right] V^{\rho}, \sigma=1, \ldots, M,
$$

with respect to arbitrary functions $U(x)=\left(U^{1}(x), \ldots, U^{N}(x)\right)$ and $V(x)=\left(V^{1}(x), \ldots, V^{N}(x)\right)$.

Suppose also that the PDE system (1) has a point symmetry (3) with an infinitesimal generator (4), then the symmetry components $V^{\rho}=\hat{\eta}^{\rho}[U]$ arise from the solutions $\hat{\eta}^{\rho}[u]$ of the symmetry determining equations, i.e., the linearized system:

$$
L_{\rho}^{\sigma}[u] \hat{\eta}^{\rho}[u]=0, \sigma=1, \ldots, M
$$

for any solution $u(x)$ of (1).

The adjoint operator (adjoint Fréchet derivative) $L^{*}[U]$ connected with the PDE system (1) is obtained formally through integration by parts and is given by:

$$
L_{\rho}^{* \sigma}[U] W_{\sigma}=\frac{\partial G_{\sigma}[U]}{\partial U^{\rho}} W_{\sigma}-D_{i}\left(\frac{\partial G_{\sigma}[U]}{\partial U_{i}^{\rho}} W_{\sigma}\right)+\cdots+(-1)^{k} D_{i_{1}} \cdots D_{i_{k}}\left(\frac{\partial G_{\sigma}[U]}{\partial U_{i_{1} \cdots i_{k}}^{\rho}} W_{\sigma}\right), \rho=1, \ldots, N,
$$

with respect to an arbitrary function $W(x)=\left(W_{1}(x), \ldots, W_{N}(x)\right)$.

For any solution $U(x)=u(x)$ of (1), a set of functions $\left\{\omega_{\sigma}[u]\right\}_{\sigma=1}^{N}$ that satisfies the adjoint linearized system:

$$
L_{\rho}^{* \sigma}[u] \omega_{\sigma}[u]=0, \rho=1, \ldots, N,
$$

is named an adjoint symmetry [21,22] of System (1). As a rule, the adjoint system is a subset of the linear determining equations for local multipliers in the direct construction method.

Theorem 1. For a PDE system (1), any pair consisting of a symmetry components $\hat{\eta}^{\rho}[u]$ and an adjoint symmetry $\left\{\omega_{\sigma}[u]\right\}_{\sigma=1}^{N}$, with solution $u(x)$ replaced by an arbitrary function $U(x)$, satisfies the conservation laws' identity $[5,7,20]$ :

$$
W_{\sigma} L_{\rho}^{\sigma}[U] \hat{\eta}^{\rho}-\hat{\eta}^{\rho} L_{\rho}^{* \sigma}[U] W_{\sigma}=D_{i} \psi^{i}[U]
$$

with:

$$
\psi^{i}[U]=\sum_{p=0}^{k-1} \sum_{q=0}^{k-p-1}(-1)^{q}\left(D_{i_{1}} \cdots D_{i_{p}} \hat{\eta}^{\rho}\right) \times D_{j_{1}} \cdots D_{j_{q}}\left(\omega_{\sigma} \frac{\partial P^{\sigma}[U]}{\partial U_{j_{1} \cdots j_{q} i_{1} \cdots i_{p}}^{\rho}}\right),
$$

where $1 \leq j_{1} \leq \cdots \leq j_{q} \leq i \leq i_{1} \leq \cdots \leq i_{p} \leq n$.

If $U(x)=u(x)$ in (18) and (19), we obtain a local conservation law $D_{i} \psi^{i}[u]=0$ of the PDE system (1), resulting from a pair of symmetry and adjoint symmetry for (1).

Recently, Ma [23] has utilized the pairs of symmetries and adjoint symmetries for presenting conservation laws of discrete evaluation equations without a Lagrangian. 


\section{Symmetry Action on a Known Conservation Law Method}

More than a decade ago, symmetry action on a known conservation law method $[5,10]$ was introduced firstly by Bluman, Temuerchaolu and Anco. Since this method establishes a significant relationship between the symmetries and conservation laws of given DE systems without a Lagrangian, we review it in this section. In order to demonstrate the application of this method, we will introduce the process of constructing conservation laws of a nonlinear telegraph system in Section 5.3.

In symmetry action on a known conservation law method, the authors have presented two important formulas related to constructing new conservation laws for any system of PDEs from the known conservation law through the action of an invertible transformation. The first formula transforms any conservation law of a PDE system (1) to the corresponding conservation law of the system obtained under a contact transformation. When the contact transformation is a symmetry, the second formula checks a priori whether the action of a (continuous or discrete) symmetry on a conservation law of (1) yields one or more new conservation laws of (1).

\subsection{Contact Transformation Action on a Conservation Law}

Consider an invertible contact transformation $[5,10]$ acting on $\left(z, W, \partial_{z} W\right)$-space:

$$
\begin{aligned}
& x_{i}=x_{i}\left(z, W, \partial_{z} W\right), i=1, \ldots, n, \\
& U^{\sigma}=U^{\sigma}\left(z, W, \partial_{z} W\right), \sigma=1, \ldots, N, \\
& U_{i}^{\sigma}=U_{i}^{\sigma}\left(z, W, \partial_{z} W\right)
\end{aligned}
$$

with $U_{i}^{\sigma}$ given by the formula:

$$
\left[\begin{array}{c}
U_{1}^{\sigma} \\
U_{2}^{\sigma} \\
\cdot \\
\cdot \\
\cdot \\
U_{n}^{\sigma}
\end{array}\right]=\mathrm{A}^{-1}[W]\left[\begin{array}{c}
\tilde{D}_{1} U_{1}^{\sigma} \\
\tilde{D}_{2} U_{2}^{\sigma} \\
\cdot \\
\cdot \\
\cdot \\
\tilde{D}_{n} U_{n}^{\sigma}
\end{array}\right]
$$

with respect to the inverse of the Jacobian matrix of $x_{i}(i=1,2, \cdots, n)$ in (20) given by:

$$
\mathrm{A}[W]=\left[\begin{array}{cccc}
\tilde{D}_{1} x_{1} & \tilde{D}_{1} x_{2} & \cdots & \tilde{D}_{1} x_{n} \\
\tilde{D}_{2} x_{1} & \tilde{D}_{2} x_{2} & \cdots & \tilde{D}_{2} x_{n} \\
\cdot & \cdot & \cdots & \cdot \\
\cdot & \cdot & \cdots & \cdot \\
\cdot & \cdot & \cdots & \cdot \\
\tilde{D}_{n} x_{1} & \tilde{D}_{n} x_{2} & \cdots & \tilde{D}_{n} x_{n}
\end{array}\right]
$$

and the total derivative operators:

$$
\tilde{D}_{i}=\frac{\partial}{\partial z_{i}}+W_{i}^{\sigma} \frac{\partial}{\partial W^{\sigma}}+W_{i j}^{\sigma} \frac{\partial}{\partial W_{j}^{\sigma}}+\cdots+W_{i i_{1} i_{2} \cdots i_{l}}^{\sigma} \frac{\partial}{\partial W_{i_{1} i_{2} \cdots i_{l}}^{\sigma}}+\cdots
$$

in terms of the independent variables $z_{i}$. Here, $W=\left(W^{1}, \ldots, W^{N}\right)$ expresses arbitrary functions of $z=\left(z_{1}, \ldots, z_{n}\right) ; W_{i}^{\sigma}=\partial W^{\sigma} / \partial z_{i}, W_{i j}^{\sigma}=\partial^{2} W^{\sigma} / \partial z_{i} \partial z_{j}$, etc.; and $\partial_{z}^{k} W$ denotes all $k$-th-order partial derivatives of $W^{\sigma}$ with respect to $z_{i}$. 
The contact transformation (20) naturally extends to the $\left(z, W, \partial_{z} W, \ldots, \partial_{z}^{k} W\right)$-space:

$$
\begin{aligned}
& x_{i}=x_{i}\left(z, W, \partial_{z} W\right), i=1, \ldots, n, \\
& U^{\sigma}=U^{\sigma}\left(z, W, \partial_{z} W\right), \sigma=1, \ldots, N, \\
& U_{i}^{\sigma}=U_{i}^{\sigma}\left(z, W, \partial_{z} W\right), \\
& \cdot \\
& \cdot \\
& \cdot \\
& U_{i_{1} \cdots i_{k}}^{\sigma}=U_{i_{1} \cdots i_{k}}^{\sigma}\left(z, W, \partial_{z} W, \ldots, \partial_{z}^{k} W\right) .
\end{aligned}
$$

Through a contact transformation (20), a function $G_{\alpha}[U]$ is transformed to some function $H_{\alpha}[W]$. Especially,

$$
H_{\alpha}[W]=G_{\alpha}[U]
$$

after the coordinates of $G_{\alpha}[U]$ are represented in regard to (24). If $U^{\sigma}=u^{\sigma}(x)$ solves PDE System (1), then correspondingly, $W^{\sigma}=w^{\sigma}(z)$ solves PDE system:

$$
H_{\alpha}[w]=0,
$$

involving dependent variables $w^{\sigma}(z)$ and independent variables $z_{i}$. The following theorem and its proof appear in $[5,10]$.

Theorem 2. Suppose (10) is a conservation law of PDE System (1). Through the contact transformation (20), there exist differentiable functions $\left\{\Psi^{i}[W]\right\}_{i=1}^{n}$ such that the formula:

$$
J[W] D_{i} \Phi^{i}[U]=\tilde{D}_{i} \Psi^{i}[W]
$$

holds, where $\Psi^{i}[W]$ is given explicitly in light of the determinant obtained through replacing the $i$-th column of the Jacobian determinant (see (22)):

$$
J[W]=\frac{D\left(x_{1}, \ldots, x_{n}\right)}{D\left(z_{1}, \ldots, z_{n}\right)}=\operatorname{det} A[W]
$$

by $\left[\Phi^{1}[U], \Phi^{2}[U], \ldots, \Phi^{n}[U]\right]^{\top}$, i.e.,

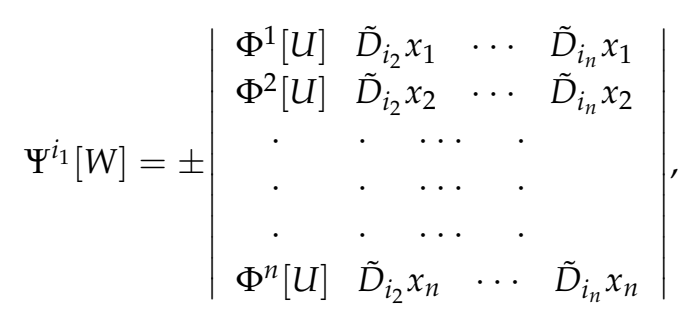

where $\left(i_{1}, i_{2}, \ldots, i_{n}\right)$ represents all cyclic permutations of the indices $1,2, \ldots, n$ and the " \pm " is chosen according to $\left(i_{1}, i_{2}, \ldots, i_{n}\right)=(1,2, \ldots, n),(2,3, \ldots, 1), \ldots,(n, 1, \ldots, n-1)$ being alternately even and odd.

From Theorem 2 above, one easily obtains the next important result.

Corollary 1. Through a contact transformation (20), a conservation law (10) for PDE System (1) is transformed to the conservation law:

$$
\tilde{D}_{i} \Psi^{i}[w]=0
$$

for PDE system (26) with fluxes $\Psi^{i}[w]$ given by (29) for any $W=w(z)$ solving PDE System (26). 


\subsection{Symmetry Action on a Conservation Law}

In this subsection, we show that the action of a symmetry on a conservation law of PDE System (1) could yield new conservation laws of (1) when the contact transformation (20) is a symmetry. Since a symmetry of (1) leaves invariant the solution manifold of (1), there exist specific functions $A_{\alpha}^{\beta}[W]$ so that (25) is of the form:

$$
G_{\alpha}[U]=H_{\alpha}[W]=A_{\alpha}^{\beta}[W] G_{\beta}[W] .
$$

Therefore, through Formulas (27) and (29), one obtains:

Corollary 2. If (20) is a symmetry of PDE System (1), then the local conservation law (10) of System (1) is transformed to a conservation law:

$$
D_{i} \Psi^{i}[u]=0
$$

of (1) with fluxes $\Psi^{i}[u]$ where $\Psi^{i}[W]$ is given by (29).

Corollary 2 explains that a symmetry (20) acting on a known conservation law (10) of PDE System (1) holds the conservation law (32) of (1) with the help of Formula (27). Now, through the next theorem $[5,10]$, one can check a priori whether or not the action of a symmetry (20) on a conservation law (10) yields new conservation laws (32) of (1).

Theorem 3. Suppose (20) is a symmetry of (1). If $\left\{\Lambda^{\alpha}[U]\right\}$ is a set of multipliers for a conservation law of PDE System (1) with fluxes $\Phi^{i}[u]$, then:

$$
\hat{\Lambda}^{\beta}[W] G_{\beta}[W]=\tilde{D}_{i} \Psi^{i}[W]
$$

where:

$$
\hat{\Lambda}^{\beta}[W]=J[W] A_{\alpha}^{\beta}[W] \Lambda^{\alpha}[U], \beta=1, \ldots, M,
$$

with $U=U(z, W)$ and its derivatives given by the transformation (24). In (33), $\Psi i[W]$ is given by (29), and in (34), $J[W]$ and $A_{\alpha}^{\beta}[W]$ arise from (28) and (31), respectively.

Corollary 3. If $\left\{\Lambda^{\alpha}[U]\right\}$ is a set of multipliers of (1) admitting (20), then $\left\{\hat{\Lambda}^{\beta}[U]\right\}$ yields a set of multipliers of (1) where $\hat{\Lambda}^{\beta}[U]$ is given by (34) after replacing $z_{i}$ by $x_{i}, W^{\sigma}$ by $U^{\sigma}, W_{i}^{\sigma}$ by $U_{i}^{\sigma}$, etc.

Suppose contact transformation is a one-parameter $(\varepsilon)$ Lie group of point transformation $[2,24]$, then one has:

$$
\begin{aligned}
& x_{i}=z_{i}+\varepsilon \xi_{i}\left(z, W, \partial_{z} W\right)+O\left(\varepsilon^{2}\right), i=1, \ldots, n \\
& U^{\sigma}=W^{\sigma}+\varepsilon \eta^{\sigma}\left(z, W, \partial_{z} W\right)+O\left(\varepsilon^{2}\right), \sigma=1, \ldots, N
\end{aligned}
$$

The extended infinitesimal generator $\tilde{X}$ associated with (35) can be written as:

$$
\tilde{X}=\xi_{j}[W] \frac{\partial}{\partial z_{j}}+\eta^{\sigma}[W] \frac{\partial}{\partial W^{\sigma}}+\eta_{i}^{\sigma}[W] \frac{\partial}{\partial W_{i}^{\sigma}}+\cdots+\eta_{i_{1} \cdots i_{k}}^{\sigma}[W] \frac{\partial}{\partial W_{i_{1} \cdots i_{k}}^{\sigma}}+\cdots
$$

with:

$$
\begin{aligned}
& \eta_{i}^{\sigma}[W]=\tilde{D}_{i} \eta^{\sigma}[W]-\left(\tilde{D}_{i} \xi_{j}[W]\right) W_{j}^{\sigma}, i=1, \cdots, n, \\
& \eta_{i_{1} \cdots i_{k}}^{\sigma}[W]=\tilde{D}_{i_{k}} \eta_{i_{1} \cdots i_{k-1}}^{\sigma}[W]-\left(\tilde{D}_{i_{k}} \xi_{j}[W]\right) W_{i_{1} \cdots i_{k-1}}^{\sigma}, \sigma=1, \cdots, N,
\end{aligned}
$$

where $i_{l}=1, \cdots, n$ for $l=1, \ldots, k, k \geqslant 2$. In terms of (36), one has $x_{i}=\exp (\varepsilon \tilde{X}) z_{i}, U^{\sigma}=$ $\exp (\varepsilon \tilde{X}) W^{\sigma}, \quad U_{i}^{\sigma}=\exp (\varepsilon \tilde{X}) W_{i}^{\sigma}, \ldots$. Let $X=\xi_{j}[U] \frac{\partial}{\partial x_{j}}+\eta^{\sigma}[U] \frac{\partial}{\partial U^{\sigma}}+\eta_{i}^{\sigma}[U] \frac{\partial}{\partial U_{i}^{\sigma}}+\cdots+$ $\eta_{i_{1} \cdots i_{k}}^{\sigma}[U] \frac{\partial}{\partial U_{i_{1} \cdots i_{k}}^{\sigma}}+\cdots$.

Now, suppose (35) is a point symmetry of (1). Then, one has an important result:

$$
X G_{\alpha}[U]=a_{\alpha}^{\beta}[U] G_{\beta}[U]
$$


for some functions $a_{\alpha}^{\beta}[U]$. Consequently, it is easy to see that:

$$
X^{k} G_{\alpha}[U]=\left(a_{\alpha}^{\beta}[U]_{k}\right) G_{\beta}[U]
$$

where $a_{\alpha}^{\beta}[U]_{1}=a_{\alpha}^{\beta}[U]$ and, for $k \geqslant 1, a_{\alpha}^{\beta}[U]_{k+1}=X a_{\alpha}^{\beta}[U]_{k}+a_{\alpha}^{\gamma}[U]_{k} a_{\gamma}^{\beta}[U]$.

Now, let two determinants:

$$
J[U ; \varepsilon]=\left|\begin{array}{cccc}
D_{1}\left(e^{\varepsilon X} x_{1}\right) & D_{1}\left(e^{\varepsilon X} x_{2}\right) & \cdots & D_{1}\left(e^{\varepsilon X} x_{n}\right) \\
D_{2}\left(e^{\varepsilon X} x_{1}\right) & D_{2}\left(e^{\varepsilon X} x_{2}\right) & \cdots & D_{2}\left(e^{\varepsilon X} x_{n}\right) \\
\cdot & \cdot & \cdots & \cdot \\
\cdot & \cdot & \cdots & \cdot \\
\cdot & \cdot & \cdots & \cdot \\
D_{n}\left(e^{\varepsilon X} x_{1}\right) & D_{n}\left(e^{\varepsilon X} x_{2}\right) & \cdots & D_{n}\left(e^{\varepsilon X} x_{n}\right)
\end{array}\right|
$$

and:

$$
\Psi^{i_{1}}[U ; \varepsilon]= \pm\left|\begin{array}{cccc}
e^{\varepsilon X} \Phi^{1}[U] & e^{\varepsilon X} \Phi^{2}[U] & \cdots & e^{\varepsilon X} \Phi^{n}[U] \\
D_{i_{2}}\left(e^{\varepsilon X} x_{1}\right) & D_{i_{2}}\left(e^{\varepsilon X} x_{2}\right) & \cdots & D_{i_{2}}\left(e^{\varepsilon X} x_{n}\right) \\
\cdot & \cdot & \cdots & \cdot \\
\cdot & \cdot & \cdots & \cdot \\
\cdot & \cdot & \cdots & \cdot \\
D_{i_{n}}\left(e^{\varepsilon X} x_{1}\right) & D_{i_{n}}\left(e^{\varepsilon X} x_{2}\right) & \cdots & D_{i_{n}}\left(e^{\varepsilon X} x_{n}\right)
\end{array}\right| .
$$

The following theorem and its proof appear in $[5,10]$.

Theorem 4. Suppose (35) is a point symmetry of PDE System (1) and $\left\{\Lambda^{\alpha}[U]\right\}$ is a set of multipliers for a conservation law of PDE system (1) with fluxes $\left\{\Phi^{j}[u]\right\}$. Suppose $J[U ; \varepsilon]$ and $\Psi^{i}[U ; \varepsilon]$ are expressed by (40) and (41), respectively. Then,

$$
\hat{\Lambda}^{\beta}[U]_{p}=\left.\sum_{n+k+l=p} \frac{1}{n ! k ! l !}\left(a_{\alpha}^{\beta}[U]_{l}\right)\left(X^{k} \Lambda^{\alpha}[U]\right) \frac{d^{n}}{d \varepsilon^{n}} J[U ; \varepsilon]\right|_{\varepsilon=0}, \beta=1, \ldots, M,
$$

defines sets of multipliers for conservation laws of (1) with fluxes given by:

$$
\Psi^{i}[u]_{p}=\left.\frac{1}{p !} \frac{d^{p}}{d \varepsilon^{p}} \Psi^{i}[u ; \varepsilon]\right|_{\varepsilon=0}
$$

for $p=0,1,2, \ldots ; \hat{\Lambda}^{\beta}[U]_{0}=\Lambda^{\beta}[U], \Psi^{i}[u]_{0}=\Phi^{i}[u], a_{\alpha}^{\beta}[U]_{0}=\delta_{\alpha \beta}$.

If PDE System (1) involves $x=\left(x_{1}, x_{2}\right)=(t, x)$ and $u=\left(u^{1}, u^{2}\right)=(u, v)$, one obtains:

$$
\begin{aligned}
& X=\tau(x, t, u, v) \frac{\partial}{\partial t}+\xi(x, t, u, v) \frac{\partial}{\partial x}+\eta(x, t, u, v) \frac{\partial}{\partial u}+\phi(x, t, u, v) \frac{\partial}{\partial v}+\cdots, \\
& D_{t}=\frac{\partial}{\partial t}+u_{t} \frac{\partial}{\partial u}+v_{t} \frac{\partial}{\partial v}, \quad D_{x}=\frac{\partial}{\partial x}+u_{x} \frac{\partial}{\partial u}+v_{x} \frac{\partial}{\partial v} .
\end{aligned}
$$

Thus, for $p=1$, one has:

$$
\begin{gathered}
\hat{\Lambda}^{\alpha}[U]_{1}=\left(D_{t} \tau+D_{x} \xi\right) \Lambda^{\alpha}[U]+X \Lambda^{\alpha}[U]+a_{\beta}^{\alpha}[U] \Lambda^{\beta}[U], \\
\Psi^{1}[U]_{1}=X \Phi^{1}[U]+\Phi^{1}[U] D_{x} \xi-\Phi^{2}[U] D_{x} \tau \\
\Psi^{2}[U]_{1}=X \Phi^{2}[U]+\Phi^{2}[U] D_{t} \tau-\Phi^{1}[U] D_{t} \xi
\end{gathered}
$$

in terms of multipliers $\left\{\Lambda^{\alpha}[U]\right\}$ for a known conservation law with fluxes $\Phi^{1}[u]$ and $\Phi^{2}[u]$. 


\section{Conservation Laws for a Nonlinear Telegraph System}

For the sequel, $x_{1}=t, x_{2}=x, u^{1}=u(x, t), u^{2}=v(x, t), U^{1}=U(x, t), U^{2}=V(x, t), z_{1}=\tilde{t}, z_{2}=$ $\tilde{x}, w^{1}=\tilde{u}(\tilde{x}, \tilde{t}), w^{2}=\tilde{v}(\tilde{x}, \tilde{t})$ and $W^{1}=\tilde{U}(\tilde{x}, \tilde{t}), W^{2}=\tilde{V}(\tilde{x}, \tilde{t}), W=\left(W^{1}, W^{2}\right)$.

To illustrate the above approaches, we consider as an example nonlinear telegraph (NLT) systems [10,25-27] of the form:

$$
G_{1}[u, v]=v_{t}-P(u) u_{x}-Q(u)=0, G_{2}[u, v]=u_{t}-v_{x}=0,
$$

where $P(u)$ and $Q(u)$ are arbitrary functions with respect to $u$.

Suppose $P(u)=\sec ^{2} u, Q(u)=\tan u$ [26]. Then, (47) becomes:

$$
G_{1}[u, v]=v_{t}-\left(\sec ^{2} u\right) u_{x}-\tan u=0, G_{2}[u, v]=u_{t}-v_{x}=0 .
$$

The NLT system (48) admits three translations [27]:

$$
\begin{aligned}
& t=\tilde{t}+\varepsilon, x=\tilde{x}, u=\tilde{u}, v=\tilde{v}, \\
& t=\tilde{t}, x=\tilde{x}, u=\tilde{u}, v=\tilde{v}+\varepsilon, \\
& t=\tilde{t}, x=\tilde{x}+\varepsilon, u=\tilde{u}, v=\tilde{v}
\end{aligned}
$$

as well as the one-parameter Lie group of point transformation [26]:

$$
t^{*}=t \cos \varepsilon-v \sin \varepsilon, x^{*}=x+\ln |\cos (u+\varepsilon) / \cos u|, u^{*}=u+\varepsilon, v^{*}=v \cos \varepsilon+t \sin \varepsilon,
$$

yielding four point symmetries with the infinitesimal:

$$
\xi=-C_{1} \tan u+C_{3}, \tau=-C_{1} v+C_{2}, \eta=C_{1}, \zeta=C_{1} t+C_{4} .
$$

\subsection{Direct Construction Method for NLT Systems (48)}

From the determining system (12) for multipliers, one obtains the five zeroth-order multipliers $\Lambda^{1}=\Lambda^{1}\left(x_{1}, x_{2}, U^{1}, U^{2}\right)$ and $\Lambda^{2}=\Lambda^{2}\left(x_{1}, x_{2}, U^{1}, U^{2}\right)$ for the NLT system (48), given by:

$$
\begin{gathered}
\left(\Lambda_{1}^{1}, \Lambda_{1}^{2}\right)=\left(e^{x}\left(2 x+t^{2}+V^{2}-2 \ln |\cos U|\right), 2 e^{x}(t+V \tan U)\right), \\
\left(\Lambda_{2}^{1}, \Lambda_{2}^{2}\right)=\left(t e^{x}, e^{x}\right),\left(\Lambda_{3}^{1}, \Lambda_{3}^{2}\right)=\left(V e^{x}, e^{x} \tan U\right),\left(\Lambda_{4}^{1}, \Lambda_{4}^{2}\right)=(0,1),\left(\Lambda_{5}^{1}, \Lambda_{5}^{2}\right)=\left(e^{x}, 0\right) .
\end{gathered}
$$

Then, from (9) and (52), one has the five fluxes of (48) satisfying $\frac{\partial}{\partial t} \Phi_{i}^{t}[u, v]+$ $\frac{\partial}{\partial x} \Phi_{i}^{x}[u, v]=0, i=1,2, \ldots, 5$ with:

$$
\begin{aligned}
\left(\Phi_{1}^{t}, \Phi_{1}^{x}\right) & =\left(e^{x}\left[2 t u+\frac{1}{3} v^{3}+v\left(2+t^{2}+2 x-2 \ln |\cos u|\right)\right]\right. \\
& \left.e^{x}\left[\left(-v^{2}-t^{2}-2 x+2(1+\ln |\cos u|)\right) \tan u-2(t v+u)\right]\right) ; \\
\left(\Phi_{2}^{t}, \Phi_{2}^{x}\right) & =\left(e^{x}(t v+u),-e^{x}(v+t \tan u)\right) ; \\
\left(\Phi_{3}^{t}, \Phi_{3}^{x}\right) & =\left(e^{x}\left(\frac{1}{2} v^{2}-\ln |\cos u|\right),-v e^{x} \tan u\right) ; \\
\left(\Phi_{4}^{t}, \Phi_{4}^{x}\right) & =(u,-v) ; \\
\left(\Phi_{5}^{t}, \Phi_{5}^{x}\right) & =\left(e^{x} v,-e^{x} \tan u\right) .
\end{aligned}
$$




\subsection{SA Method for NLT Systems (48)}

The conservation laws for (48) are now derived by using the SA method. From (14) and (16), one obtains the linearizing operator:

$$
L=\left[\begin{array}{cc}
-\sec ^{2} u\left(1+2 \tan u u_{x}+D_{x}\right) & D_{t} \\
D_{t} & -D_{x}
\end{array}\right]
$$

and the adjoint linearizing operator:

$$
L^{*}=\left[\begin{array}{cc}
\sec ^{2} u\left(D_{x}-1\right) & -D_{t} \\
-D_{t} & D_{x}
\end{array}\right] .
$$

The NLT system (48) has four point symmetries (51) with infinitesimal generators:

$$
X_{1}=\frac{\partial}{\partial t}, X_{2}=\frac{\partial}{\partial x}, X_{3}=\frac{\partial}{\partial v}, X_{4}=-v \frac{\partial}{\partial t}-\tan u \frac{\partial}{\partial x}+\frac{\partial}{\partial u}+t \frac{\partial}{\partial v} .
$$

Using (7), the corresponding four sets of $\hat{\eta}^{\rho}[u]$ in characteristic form are given by:

$$
\begin{aligned}
& X_{1} \rightarrow \hat{\eta}^{1}=\left(\hat{\eta}_{1}^{1}, \hat{\eta}_{2}^{1}\right)=\left(-u_{t},-v_{t}\right), \quad X_{2} \rightarrow \hat{\eta}^{2}=\left(\hat{\eta}_{1}^{2}, \hat{\eta}_{2}^{2}\right)=\left(-u_{x},-v_{x}\right), \\
& X_{3} \rightarrow \hat{\eta}^{3}=\left(\hat{\eta}_{1}^{3}, \hat{\eta}_{2}^{3}\right)=(0,1), \quad X_{4} \rightarrow \hat{\eta}^{4}=\left(\hat{\eta}_{1}^{4}, \hat{\eta}_{2}^{4}\right)=\left(1+v u_{t}+\tan u u_{x}, t+v v_{t}+\tan u v_{x}\right),
\end{aligned}
$$

these can be obtained from the combination of the linearizing system (15) and linearizing operator (54).

From (17) and (55), one obtains the adjoint linearized system:

$$
L^{*} \omega_{\sigma}=\left[\begin{array}{cc}
\sec ^{2} u\left(D_{x}-1\right) & -D_{t} \\
-D_{t} & D_{x}
\end{array}\right]\left[\begin{array}{c}
\omega_{i}^{1} \\
\omega_{i}^{2}
\end{array}\right]=\left[\begin{array}{l}
0 \\
0
\end{array}\right]
$$

for functions $\omega_{i}^{1}=\omega_{i}^{1}(x, t, u, v)$ and $\omega_{i}^{2}=\omega_{i}^{2}(x, t, u, v)$. Solving (58), one obtains the following five solutions (adjoint symmetries):

$$
\begin{aligned}
& \left(\omega_{1}^{1}, \omega_{1}^{2}\right)=\left(e^{x}\left(2 x+t^{2}+v^{2}-2 \ln |\cos u|\right), 2 e^{x}(t+v \tan u)\right),\left(\omega_{2}^{1}, \omega_{2}^{2}\right)=\left(t e^{x}, e^{x}\right), \\
& \left(\omega_{3}^{1}, \omega_{3}^{2}\right)=\left(v e^{x}, e^{x} \tan u\right), \quad\left(\omega_{4}^{1}, \omega_{4}^{2}\right)=(0,1),\left(\omega_{5}^{1}, \omega_{5}^{2}\right)=\left(e^{x}, 0\right) .
\end{aligned}
$$

Now, after substituting $\hat{\eta}^{2}=\left(\hat{\eta}_{1}^{2}, \hat{\eta}_{2}^{2}\right)$ in (57) and $\left(\omega_{1}^{1}, \omega_{1}^{2}\right)$ in (59) into (18) and after cancellation of trivial conservation terms, one yields the conservation law with fluxes:

$$
\begin{aligned}
& \psi_{1}^{t}=e^{x}\left[2 t u+\frac{1}{3} v^{3}+v\left(2+t^{2}+2 x-2 \ln |\cos u|\right)\right] \\
& \psi_{1}^{x}=e^{x}\left[\left(-v^{2}-t^{2}-2 x+2(1+\ln |\cos u|)\right) \tan u-2(t v+u)\right]
\end{aligned}
$$

Using all pairs $\left\langle\hat{\eta}^{\rho},\left(\omega_{\sigma}^{1}, \omega_{\sigma}^{2}\right)\right\rangle(\rho=1,2,3,4, \sigma=1,2,3,4,5)$ from (57) and (59), one obtains another three conservation laws of (48), with flux components:

$$
\begin{aligned}
& \left\langle\hat{\eta}^{1},\left(\omega_{2}^{1}, \omega_{2}^{2}\right)\right\rangle,\left\langle\hat{\eta}^{2},\left(\omega_{5}^{1}, \omega_{5}^{2}\right)\right\rangle,\left\langle\hat{\eta}^{3},\left(\omega_{3}^{1}, \omega_{3}^{2}\right)\right\rangle:\left(\psi_{2}^{t}, \psi_{2}^{x}\right)=\left[e^{x} v,-e^{x} \tan u\right] ; \\
& \left\langle\hat{\eta}^{1},\left(\omega_{1}^{1}, \omega_{1}^{2}\right)\right\rangle,\left\langle\hat{\eta}^{2},\left(\omega_{2}^{1}, \omega_{2}^{2}\right)\right\rangle,\left\langle\hat{\eta}^{4},\left(\omega_{3}^{1}, \omega_{3}^{2}\right)\right\rangle:\left(\psi_{3}^{t}, \psi_{3}^{x}\right)=\left[e^{x}(t v+u),-e^{x}(v+t \tan u)\right] ; \\
& \left\langle\hat{\eta}^{3},\left(\omega_{1}^{1}, \omega_{1}^{2}\right)\right\rangle,\left\langle\hat{\eta}^{2},\left(\omega_{3}^{1}, \omega_{3}^{2}\right)\right\rangle,\left\langle\hat{\eta}^{4},\left(\omega_{2}^{1}, \omega_{2}^{2}\right)\right\rangle:\left(\psi_{4}^{t}, \psi_{4}^{x}\right)=\left[e^{x}\left(\frac{1}{2} v^{2}-\ln |\cos u|\right),-v e^{x} \tan u\right] .
\end{aligned}
$$

\subsection{Symmetry Action on Known Conservation Laws for NLT System (48)}

One can show that NLT System (48) has a conservation law with fluxes (60), and it produces a set of multipliers (52a):

$$
\Lambda^{1}[U]=e^{x}\left(2 x+t^{2}+V^{2}-2 \ln |\cos U|\right), \Lambda^{2}[U]=2 e^{x}(t+V \tan U) .
$$


The NLT system (48) admits three continuous translation symmetries (49) and the point symmetry with infinitesimal generator:

$$
X=-V \frac{\partial}{\partial t}-\tan U \frac{\partial}{\partial x}+\frac{\partial}{\partial U}+t \frac{\partial}{\partial V}
$$

resulting from (51).

(I) Under the translation symmetry (49):

Under the symmetry (49), one has $J[W]=1, H_{1}[W]=G_{1}[U]=G_{1}[W], H_{2}[W]=G_{2}[U]=G_{2}[W]$. Thus, in (31), one yields $A_{1}^{1}[W]=1, A_{2}^{2}[W]=1, A_{1}^{2}[W]=A_{2}^{1}[W]=0$. After using the translation symmetry (49a) for the known conservation law resulting from the multipliers (62) with fluxes (60), from Formula (34) and Corollary 3, one obtains:

$$
\begin{aligned}
& \hat{\Lambda}^{1}[W]=e^{\tilde{x}}\left[2 \tilde{x}+\tilde{t}^{2}+2 \varepsilon \tilde{t}+\varepsilon^{2}+\tilde{V}^{2}-2 \ln |\cos \tilde{U}|\right]=\Lambda^{1}[W]+2 \tilde{t}^{\tilde{x}} \varepsilon+e^{\tilde{x}} \varepsilon^{2} \\
& \hat{\Lambda}^{2}[W]=2 e^{\tilde{x}}(\tilde{t}+\varepsilon+\tilde{V} \tan \tilde{U})=\Lambda^{2}[W]+2 e^{\tilde{x}} \varepsilon .
\end{aligned}
$$

Then, applying the first formula (29), one obtains:

$$
\begin{aligned}
& \Psi^{1}[W]=\Phi^{1}[W]+2 e^{\tilde{x}}(\tilde{t} \tilde{V}+\tilde{U}) \varepsilon+\tilde{V} e^{\tilde{x}} \varepsilon^{2} \\
& \Psi^{2}[W]=\Phi^{2}[W]-2 e^{\tilde{x}}(\tilde{t} \tan \tilde{U}+\tilde{V}) \varepsilon-e^{\tilde{x}} \tan \tilde{U} \varepsilon^{2}
\end{aligned}
$$

Comparing (62) and (64), one sees that the $O(\varepsilon)$ terms in (64) and (65) hold new multipliers:

$$
\lambda^{1}[U]=\tilde{\Lambda}^{1}[U]_{1}=t e^{x}, \lambda^{2}[U]=\tilde{\Lambda}^{2}[U]_{1}=e^{x}
$$

and the corresponding conservation law with fluxes:

$$
\begin{aligned}
& \phi^{1}[u]=\Psi^{1}[u]_{1}=e^{x}(t v+u), \\
& \phi^{2}[u]=\Psi^{2}[u]_{1}=-e^{x}(t \tan u+v)
\end{aligned}
$$

the $O\left(\varepsilon^{2}\right)$ terms in (64) and (65) hold the new multipliers and fluxes:

$$
\tilde{\Lambda}^{1}[U]_{2}=e^{x}, \tilde{\Lambda}^{2}[U]_{2}=0, \Psi^{1}[u]_{2}=v e^{x}, \Psi^{2}[u]_{2}=-e^{x} \tan u .
$$

Similarly, after using the translation symmetry (49b) for the known conservation law resulting from the multipliers (62) with fluxes (60), Formula (34) leads to:

$$
\begin{aligned}
& \check{\Lambda}^{1}[W]=e^{\tilde{x}}\left[2 \tilde{x}+\tilde{t}^{2}+\tilde{V}^{2}+2 \varepsilon \tilde{V}+\varepsilon^{2}-2 \ln |\cos \tilde{U}|\right]=\Lambda^{1}[W]+2 \tilde{V} e^{\tilde{x}} \varepsilon+e^{\tilde{x}} \varepsilon^{2}, \\
& \check{\Lambda}^{2}[W]=2 e^{\tilde{x}}(\tilde{t}+\tilde{V} \tan \tilde{U}+\varepsilon \tan \tilde{U})=\Lambda^{2}[W]+2 e^{\tilde{x}} \tan \tilde{U} \varepsilon .
\end{aligned}
$$

Then, Formula (29) yields:

$$
\begin{aligned}
& \check{\Psi}^{1}[W]=\Phi^{1}[W]+e^{\tilde{x}}\left(\frac{1}{2} \tilde{V}^{2}-\ln |\cos \tilde{U}|\right) \varepsilon+\tilde{V} e^{\tilde{x}} \varepsilon^{2} \\
& \check{\Psi}^{2}[W]=\Phi^{2}[W]-e^{\tilde{x}} \tilde{V} \tan \tilde{U} \varepsilon-e^{\tilde{x}} \tan \tilde{U} \varepsilon^{2} .
\end{aligned}
$$

Comparing (62) and (69), one holds two conservation laws of NLT System (48). Firstly, the $O(\varepsilon)$ terms in (69) and (70) yield new multipliers:

$$
\tilde{\Lambda}^{1}[U]_{3}=V e^{x}, \tilde{\Lambda}^{2}[U]_{3}=e^{x} \tan U
$$

and fluxes:

$$
\begin{aligned}
& \Psi^{1}[u]_{3}=e^{x}\left(\frac{1}{2} v^{2}-\ln |\cos u|\right), \\
& \Psi^{2}[u]_{3}=-v e^{x} \tan u ;
\end{aligned}
$$

secondly, the $O\left(\varepsilon^{2}\right)$ terms in (69) and (70) hold also the multipliers and fluxes (68). 


\section{(II) Under the point symmetry (63):}

Under the point symmetry (63), one obtains $X G_{1}[U]=\left(V_{t}+U_{x} \sec ^{2} U+\tan U\right) G_{1}[U], X G_{2}[U]=$ $\left(V_{t}+U_{x} \sec ^{2} U\right) G_{2}[U]$, and thus, in (38), one obtains $a_{1}^{1}[U]=V_{t}+U_{x} \sec ^{2} U+\tan U, a_{1}^{2}[U]=$ $a_{2}^{1}[U]=0, a_{2}^{2}[U]=V_{t}+U_{x} \sec ^{2} U$. The admitted point symmetry (63) is used for the $O(\varepsilon)$ term conservation law with fluxes (67). Then, in (45) with $\Lambda^{1}[U]=\lambda^{1}[U]=t e^{x}, \Lambda^{2}[U]=\lambda^{2}[U]=e^{x}$ given by (66), we have $\tau=-V, \xi=-\tan U, X \lambda^{1}[U]=-e^{x}(V+t \tan U)$ and $X \lambda^{2}[U]=-e^{x} \tan U$. Therefore, here, (45) obtains a third multiplier generated by:

$$
\hat{\lambda}^{1}[U]_{1}=V e^{x}, \hat{\lambda}^{2}[U]_{1}=e^{x} \tan U .
$$

Hence, Formula (46) with $\Phi^{1}[U]=\phi^{1}[U], \Phi^{2}[U]=\phi^{2}[U]$ given by (67), obtains a conservation law for NLT System (48) with fluxes:

$$
\begin{aligned}
& \phi^{1}[u]_{1}=e^{x}\left(\frac{1}{2} v^{2}-\ln |\cos u|\right), \\
& \phi^{2}[u]_{1}=-v e^{x} \tan u,
\end{aligned}
$$

which is the same as (72).

\subsection{Ibragimov's Conservation Theorem for NLT Systems (48)}

In order to further reveal the relationship between the SA method and Ibragimov's conservation theorem in the next subsection, the construction of conservation laws of NLT System (48) is firstly viewed by using Ibragimov's conservation theorem in this subsection.

With the help of Lie symmetry generators (4), formal Lagrangian $L=\mu^{\alpha} G_{\alpha}[11,28]$ (where $\mu^{\alpha}=\mu^{\alpha}\left(x_{i}, u^{\sigma}\right)$ are new adjoint variables (multipliers)) and adjoint equation system:

$$
G_{\alpha}^{*}[u, \mu]=E_{u^{\sigma}} L=0, \alpha=1,2, \ldots, M, \sigma=1,2, \ldots, N,
$$

Ibragimov [11] proved the following so-called new conservation theorem.

Theorem 5. Every Lie point symmetry (3) with (4) of PDE System (1) holds a conservation law for PDE System (1) and adjoint equation System (75). The conserved vectors are given by the formula:

$$
T^{i}=\xi^{i} L+W^{\sigma} \frac{\delta L}{\delta u_{i}^{\sigma}}+\sum_{s \geqslant 1} D_{i_{1}} \ldots D_{i_{s}}\left(W^{\sigma}\right) \frac{\delta L}{\delta u_{i i_{1} \ldots i_{s}}^{\sigma}},
$$

where $i=1, \ldots, n, \sigma=1, \ldots, N$ and $W^{\sigma}=\eta^{\sigma}-\xi^{j} u_{j}^{\sigma}$ (characteristic functions of (7)).

We can obtain the Lagrangian of NLT System (48) with:

$$
L=\mu^{1}\left(v_{t}-\sec ^{2} u u_{x}-\tan u\right)+\mu^{2}\left(u_{t}-v_{x}\right),
$$

where $\mu^{1}=\mu^{1}(x, t, u, v)$ and $\mu^{2}=\mu^{2}(x, t, u, v)$ are two new adjoint variables. Using (75), the adjoint equation system can be obtained with the aid of formal Lagrangian (77) for NLT System (48) as:

$$
\begin{aligned}
& F_{1}^{*}=E_{u} L=\mu_{t}^{2}+\left(\mu^{1}-\mu_{x}^{1}\right) \sec ^{2} u=0, \\
& F_{2}^{*}=E_{v} L=-\mu_{t}^{1}+\mu_{x}^{2}=0 .
\end{aligned}
$$

The multipliers determining Equation (12) are connected closely with the determining equations for adjoint symmetries (17) [21,22] and the determining equations for adjoint variables $\mu^{\alpha}=\mu^{\alpha}\left(x_{i}, u^{\sigma}\right)$ 
of formal Lagrangian $L$. Thus, with the help of the multiplier determining Equation (12), one can obtain the solutions of adjoint equation System (78), which are given by:

$$
\begin{aligned}
& \left(\mu_{1}^{1}, \mu_{1}^{2}\right)=\left(e^{x}\left(2 x+t^{2}+v^{2}-2 \ln |\cos u|\right), 2 e^{x}(t+v \tan u)\right),\left(\mu_{2}^{1}, \mu_{2}^{2}\right)=\left(t e^{x}, e^{x}\right), \\
& \left(\mu_{3}^{1}, \mu_{3}^{2}\right)=\left(v e^{x}, e^{x} \tan u\right),\left(\mu_{4}^{1}, \mu_{4}^{2}\right)=(0,1),\left(\mu_{5}^{1}, \mu_{5}^{2}\right)=\left(e^{x}, 0\right) .
\end{aligned}
$$

Now, after substituting $X_{2}$ in (56) and $\left(\mu_{1}^{1}, \mu_{1}^{2}\right)$ in (79) into (76) and after cancellation of trivial conservation terms, one obtains the conservation laws with fluxes:

$$
\begin{aligned}
& T_{1}^{t}=e^{x}\left[2 t u+\frac{1}{3} v^{3}+v\left(t^{2}+2 x+2-2 \ln |\cos u|\right)\right], \\
& T_{1}^{x}=e^{x}\left[\left(-v^{2}-t^{2}-2 x+2(1+\ln |\cos u|)\right) \tan u-2(t v+u)\right] .
\end{aligned}
$$

Similarly, using the combinations $\left\langle X_{i},\left(\mu_{j}^{1}, \mu_{j}^{2}\right)\right\rangle(i=1,2,3,4, j=1,2,3,4,5)$ for (56) and (79), one obtains another three conservation laws of (48) from (76), with fluxes:

$$
\begin{aligned}
& \left\langle X_{1},\left(\mu_{2}^{1}, \mu_{2}^{2}\right)\right\rangle,\left\langle X_{2},\left(\mu_{5}^{1}, \mu_{5}^{2}\right)\right\rangle,\left\langle X_{3},\left(\mu_{3}^{1}, \mu_{3}^{2}\right)\right\rangle:\left(T_{2}^{t}, T_{2}^{x}\right)=\left[e^{x} v,-e^{x} \tan u\right] ; \\
& \left\langle X_{1},\left(\mu_{1}^{1}, \mu_{1}^{2}\right)\right\rangle,\left\langle X_{2},\left(\mu_{2}^{1}, \mu_{2}^{2}\right)\right\rangle,\left\langle X_{4},\left(\mu_{3}^{1}, \mu_{3}^{2}\right)\right\rangle:\left(T_{3}^{t}, T_{3}^{x}\right)=\left[e^{x}(t v+u),-e^{x}(v+t \tan u)\right] ; \\
& \left\langle X_{2},\left(\mu_{3}^{1}, \mu_{3}^{2}\right)\right\rangle,\left\langle X_{3},\left(\mu_{1}^{1}, \mu_{1}^{2}\right)\right\rangle,\left\langle X_{4},\left(\mu_{2}^{1}, \mu_{2}^{2}\right)\right\rangle:\left(T_{4}^{t}, T_{4}^{x}\right)=\left[e^{x}\left(\frac{1}{2} v^{2}-\ln |\cos u|\right),-v e^{x} \tan u\right] .
\end{aligned}
$$

\subsection{Comparison of These Methods for Conservation Laws}

We can calculate several conservation laws for the NLT system (48) under the direct construction method, SA method, symmetry action on a known conservation law method and Ibragimov's theorem. Although these methods are different ostensibly, the constructions of the conservation laws are related to each other virtually. Recently, Anco [12] has found that Ibragimov's conservation law formula (76) is a simple re-writing of a special situation of the bilinear skew-symmetric identity (18) using symmetries and adjoint symmetries.

The adjoint system (58) for NLT System (48) from the Fréchet derivative identity (adjoint operator) (16) is equivalent to the adjoint equation system (78) through the formal Lagrangian (77). Hence, the solutions (adjoint symmetries) (59) of adjoint System (58) and solutions (79) of adjoint equation System (78) are equal, i.e, $\left(\omega_{j}^{1}, \omega_{j}^{2}\right)=\left(\mu_{j}^{1}, \mu_{j}^{2}\right), j=1,2, \ldots, 5$. Moreover, the characteristic form (57) is given directly from symmetry components in point symmetries (51) with infinitesimal generators (56). From Table 1 , we obviously investigate the relationship between $\left(\psi_{k}^{t}, \psi_{k}^{x}\right)$ and $\left(T_{k}^{t}, T_{k}^{x}\right)$ for NLT System (48), which is as follows

$$
\left(\psi_{1}^{t}, \psi_{1}^{x}\right)=\left(T_{1}^{t}, T_{1}^{x}\right),\left(\psi_{2}^{t}, \psi_{2}^{x}\right)=\left(T_{2}^{t}, T_{2}^{x}\right),\left(\psi_{3}^{t}, \psi_{3}^{x}\right)=\left(T_{3}^{t}, T_{3}^{x}\right),\left(\psi_{4}^{t}, \psi_{4}^{x}\right)=\left(T_{4}^{t}, T_{4}^{x}\right) .
$$

The results again shows that the conservation theorem presented by Ibragimov along with some of its extensions are shown to comprise a special case of a conservation identity (18) for the SA method.

Otherwise, we see that the action of the point symmetry $X=X_{4}$ action on a multiplier $\left(\lambda^{1}, \lambda^{2}\right)=$ $\left(\omega_{3}^{1}, \omega_{3}^{2}\right)$ and its corresponding conserved quantity $\left(\phi^{1}, \phi^{2}\right)=\left(\psi_{3}^{t}, \psi_{3}^{x}\right)$ produce a new multiplier $\left(\hat{\lambda}_{1}^{1}, \hat{\lambda}_{1}^{2}\right)=\left(\omega_{2}^{1}, \omega_{2}^{2}\right)$ and its corresponding conserved quantity $\left(\phi_{1}^{1}, \phi_{1}^{2}\right)=\left(\psi_{4}^{t}, \psi_{4}^{x}\right)$ in Section 5.3 (II) with Section 5.2. This shows that the SA method and symmetry action on a known conservation law method are connected strictly to each other for calculating conservation laws in the framework of symmetry theory and multiplier algebra in this example. 
Table 1. Comparison of the symmetry/adjoint symmetry pair method (SA method) and Ibragimov's theorem for nonlinear telegraph (NLT) System (48).

\begin{tabular}{|c|c|c|c|}
\hline \multicolumn{2}{|c|}{ Symmetry/Adjoint Symmetry Pair Method } & \multicolumn{2}{|c|}{ Ibragimov's Theorem } \\
\hline $\begin{array}{l}\left\langle\hat{\eta}^{i},\left(\omega_{j}^{1}, \omega_{j}^{2}\right)\right\rangle \\
\left.\left\langle\hat{\eta}^{2},\left(\omega_{1}^{1}, \omega_{1}^{2}\right)\right\rangle\right\rangle \\
\left\langle\hat{\eta}^{1},\left(\omega_{2}^{1}, \omega_{2}^{2}\right)\right\rangle \\
\left\langle\hat{\eta}^{1},\left(\omega_{1}^{1}, \omega_{1}^{2}\right)\right\rangle \\
\left\langle\hat{\eta}^{2},\left(\omega_{2}^{1}, \omega_{2}^{2}\right)\right\rangle \\
\left.\left\langle\hat{\eta}^{2},\left(\omega_{1}^{1}, \omega_{3}^{2}\right)\right\rangle\right\rangle \\
\left\langle\hat{\eta}^{2},\left(\omega_{5}^{1}, \omega_{5}^{2}\right)\right\rangle \\
\left\langle\hat{\eta}^{3},\left(\omega_{1}^{1}, \omega_{1}^{2}\right)\right\rangle \\
\left\langle\hat{\eta}^{3},\left(\omega_{3}^{1}, \omega_{3}^{2}\right)\right\rangle \\
\left\langle\hat{\eta}^{4},\left(\omega_{2}^{1}, \omega_{2}^{2}\right)\right\rangle \\
\left\langle\hat{\eta}^{4},\left(\omega_{3}^{1}, \omega_{3}^{2}\right)\right\rangle\end{array}$ & $\begin{array}{l}\left(\psi_{k}^{t}, \psi_{k}^{x}\right) \\
\left(\psi_{1}^{t}, \psi_{1}^{x}\right) \\
\left(\psi_{2}^{t}, \psi_{2}^{x}\right) \\
\left(\psi_{3}^{t}, \psi_{3}^{x}\right) \\
\left(\psi_{3}^{t}, \psi_{3}^{x}\right) \\
\left(\psi_{4}^{t}, \psi_{4}^{x}\right) \\
\left(\psi_{2}^{t}, \psi_{2}^{x}\right) \\
\left(\psi_{4}^{t}, \psi_{4}^{x}\right) \\
\left(\psi_{2}^{t}, \psi_{2}^{x}\right) \\
\left(\psi_{4}^{t}, \psi_{4}^{x}\right) \\
\left(\psi_{3}^{t}, \psi_{3}^{x}\right)\end{array}$ & $\begin{array}{l}\left\langle X_{i},\left(\mu_{j}^{1}, \mu_{j}^{2}\right)\right\rangle \\
\left\langle X_{2},\left(\mu_{1}^{1}, \mu_{1}^{2}\right)\right\rangle \\
\left\langle X_{1},\left(\mu_{2}^{1}, \mu_{2}^{2}\right)\right\rangle \\
\left\langle X_{1},\left(\mu_{1}^{1}, \mu_{1}^{2}\right)\right\rangle \\
\left\langle X_{2},\left(\mu_{2}^{1}, \mu_{2}^{2}\right)\right\rangle \\
\left\langle X_{2},\left(\mu_{3}^{1}, \mu_{3}^{2}\right)\right\rangle \\
\left\langle X_{2},\left(\mu_{5}^{1}, \mu_{5}^{2}\right)\right\rangle \\
\left\langle X_{3},\left(\mu_{1}^{1}, \mu_{1}^{2}\right)\right\rangle \\
\left\langle X_{3},\left(\mu_{3}^{1}, \mu_{3}^{2}\right)\right\rangle \\
\left\langle X_{4},\left(\mu_{2}^{1}, \mu_{2}^{2}\right)\right\rangle \\
\left\langle X_{4},\left(\mu_{3}^{1}, \mu_{3}^{2}\right)\right\rangle\end{array}$ & $\begin{array}{l}\left(T_{k}^{t}, T_{k}^{x}\right) \\
\left(T_{1}^{t}, T_{1}^{x}\right) \\
\left(T_{2}^{t}, T_{2}^{x}\right) \\
\left(T_{3}^{t}, T_{3}^{x}\right) \\
\left(T_{3}^{t}, T_{3}^{x}\right) \\
\left(T_{4}^{t}, T_{4}^{x}\right) \\
\left(T_{2}^{t}, T_{2}^{x}\right) \\
\left(T_{4}^{t}, T_{4}^{x}\right) \\
\left(T_{2}^{t}, T_{2}^{x}\right) \\
\left(T_{4}^{t}, T_{4}^{x}\right) \\
\left(T_{3}^{t}, T_{3}^{x}\right)\end{array}$ \\
\hline
\end{tabular}

\subsection{Cheviakov's Recursion Formula for NLT (48)}

In [13], the relationships between the recursion formula (13) with symmetry action on a known conservation law method and a direct conservation construction method are discussed. Since the function $f(x)$ is only related to the independent variables, this leads to this formula being insufficient for constructing all conservation laws. We have found that if all equations of PDE System (1) are divergence expressions, the recursion formula (13) can be used in $f=f(x, u)$ with independent variables $x=\left\{x_{i}\right\}_{i=1}^{n}$ and dependent variables $u=\left\{u^{k}(x)\right\}_{k=1}^{N}$. For example, if we introduce a simple multiplier $e^{x}$ in the first equation of (48), it becomes an apparent divergence system as follows:

$$
\begin{aligned}
& \tilde{G}_{1}[u]=e^{x}\left(v_{t}-\sec ^{2} u u_{x}-\tan u\right)=D_{t}\left(v e^{x}\right)+D_{x}\left(-e^{x} \tan u\right)=0, \\
& \tilde{G}_{2}[u]=u_{t}-v_{x}=D_{t}(u)+D_{x}(-v)=0 .
\end{aligned}
$$

With the help of (12), one obtains five multipliers:

$$
\begin{aligned}
& \left(f_{1}^{1}, f_{1}^{2}\right)=\left(2 x+t^{2}+v^{2}-2 \ln |\cos u|, 2 e^{x}(t+v \tan u)\right), \\
& \left(f_{2}^{1}, f_{2}^{2}\right)=\left(t, e^{x}\right),\left(f_{3}^{1}, f_{3}^{2}\right)=\left(v, e^{x} \tan u\right),\left(f_{4}^{1}, f_{4}^{2}\right)=(0,1),\left(f_{5}^{1}, f_{5}^{2}\right)=(1,0) .
\end{aligned}
$$

Using the recursion formula (13) with $D_{i} \Xi^{i}=f(x, u) D_{i} \Psi^{i}[u]$, one obtains the first conservation law for $(48)$ with $\left(f_{1}^{1}, f_{1}^{2}\right)$ of $(84)$ given by:

$$
\begin{aligned}
& \left(2 x+t^{2}+v^{2}-2 \ln |\cos u|\right)\left[D_{t}\left(e^{x} v\right)+D_{x}\left(-e^{x} \tan u\right)\right]+2 e^{x}(t+v \tan u)\left[D_{t}(u)+D_{x}(-v)\right] \\
& =D_{t} \Xi_{1}^{1}+D_{x} \Xi_{2}^{1}=0
\end{aligned}
$$

with corresponding fluxes:

$$
\begin{aligned}
& \Xi_{1}^{1}=e^{x}\left[2 t u+\frac{v^{3}}{3}+v\left(t^{2}+2 x-2 \ln |\cos u|\right)\right], \\
& \Xi_{2}^{1}=e^{x}\left\{\left[-v^{2}-t^{2}-2 x+2(1+\ln |\cos u|)\right] \tan u-2(v t+u)\right\} .
\end{aligned}
$$

Similarly, using the pairs (13) and (84), one obtains another four conservation laws of (48), with flux components:

$$
\begin{aligned}
& \left(\Xi_{1}^{2}, \Xi_{2}^{2}\right)=\left(e^{x}(t v+u),-e^{x}(t \tan u+v)\right), \\
& \left(\Xi_{1}^{3}, \Xi_{2}^{3}\right)=\left(e^{x}\left(\frac{1}{2} v^{2}-\ln |\cos u|\right),-e^{x} v \tan u\right), \\
& \left(\Xi_{1}^{4}, \Xi_{2}^{4}\right)=(u,-v), \\
& \left(\Xi_{1}^{5}, \Xi_{2}^{5}\right)=\left(v e^{x},-e^{x} \tan u\right) .
\end{aligned}
$$


The conserved quantities (86) and (87) constructed by Cheviakov's recursion method are completely consistent with the conserved quantities (60) and (61) derived from the SA method, and the directness of the former is revealed.

Remark 1. The NTL system (48) is a potential system for NTL equation:

$$
u_{t t}-\left(\sec ^{2} u u_{x}+\tan u\right)_{x}=0,
$$

which has four multipliers $1, t, e^{x}, t e^{x}$ with corresponding fluxes respectively given by:

$$
\begin{aligned}
& \left(\Xi_{1}^{t}, \Xi_{1}^{x}\right)=\left[u_{t},-\left(\sec ^{2} u u_{x}+\tan u\right)\right] ; \\
& \left(\Xi_{2}^{t}, \Xi_{2}^{x}\right)=\left[t u_{t}-u,-t\left(\sec ^{2} u u_{x}+\tan u\right)\right] ; \\
& \left(\Xi_{3}^{t}, \Xi_{3}^{x}\right)=\left[e^{x} u_{t},-e^{x} \sec ^{2} u u_{x}\right] ; \\
& \left(\Xi_{4}^{t}, \Xi_{4}^{x}\right)=\left[e^{x}\left(t u_{t}-u\right),-t e^{x} \sec ^{2} u u_{x}\right]
\end{aligned}
$$

under the recursion formula (13). If one inserts a potential variable $v$ through the first conservation law in (89), one immediately gets the original system (48). Meanwhile, the point symmetry (63) is a potential symmetry of (88).

\section{Conclusions}

In this work, we have summarized how to calculate conservation laws from the direct construction method, SA method, symmetry action on a known conservation law method, Ibragimov's conservation theorem and a recursion formula when a symmetry transformation is admitted by a given PDE system. The direct method yields all conservation laws resulting from the corresponding multipliers for a PDE system written in Cauchy-Kovalevskaya form. The SA method uses pairs consisting of symmetries and adjoint symmetries of an adjoint system to generate conservation laws from a well-known Fréchet derivative identity. Here, the set of adjoint symmetries is a subset of the set of multipliers. Under the action of the symmetry transformation on the set of multipliers holding a known conservation law, one can determine a priori whether a new conservation law is calculated. Ibragimov's conservation theorem utilizes pairs composed by symmetries with differential substitutions for the formal Lagrangian by means of the Euler operator, where the set of differential substitutions is also a subset of the set of multipliers. Therefore, any conservation law of a PDE system produces a corresponding set of multipliers, and the starting point for utilizing our study can be the conservation law itself, as illustrated in our example.

In addition, a comparison is made between the SA method, symmetry action on a known conservation law method and Ibragimov's conservation theorem for conservation laws admitted by nonlinear telegraph systems, which verifies that Ibragimov's conservation theorem is equivalent to the SA method, and the first two methods are connected strictly to each other for constructing conservation laws in the framework of symmetry theory and multiplier algebra. We can also see that if all equations of the PDE system are divergence expressions, the recursion formula (13) can be used for $f=f(x, u)$ with independent variables $x=\left\{x_{i}\right\}_{i=1}^{n}$ and dependent variables $u=\left\{u^{k}(x)\right\}_{k=1}^{N}$. The direct method is a very complete method. It is important to reiterate that conservation laws arise from multipliers for PDEs, and all local conservation laws constructed by the direct construction method contain the conservation laws derived from all other methods.

Author Contributions: All the authors contributed equally to the work. All authors read and approved the final manuscript.

Acknowledgments: This research was funded by the National Natural Science Foundation of China (No. 11661034), the College Scientific Research Project of Inner Mongolia (No. NJZZ16279), the "321 Talents Engineering in the New Century" of Inner Mongolia, the Program for Young Talents of Science and Technology in Universities of Inner Mongolia Autonomous Region (No. NJYT-17-A23), the Youth Innovative Talents Training Program of Inner Mongolia and the Program for Innovative Research Team of Hohhot University for Nationalities (No. CXTD1402). The authors wish to express their cordial thanks to the reviewers for the constructive suggestions. 
Conflicts of Interest: The authors declare no conflict of interest.

\section{References}

1. Noether, E. Invariante Variationsprobleme. Gott. Nachr. 1918, 2, 235-257.

2. Bluman, G.W.; Kumei, S. Symmetries and Differential Equations; Springer: Heidelberg/Berlin, Germany, 1989.

3. Bluman, G.W.; Anco, S.C. Symmetry and Integration Methods for Differential Equations; Springer: Heidelberg/Berlin, Germany, 2002.

4. Olver, P.J. Applications of Lie Groups to Differential Equations; Springer: Heidelberg/Berlin, Germany, 1993.

5. Bluman, G.W.; Cheviakov, A.F.; Anco, S.C. Applications of Symmetry Methods to Partial Differential Equations; Springer: Heidelberg/Berlin, Germany, 2010.

6. Anco, S.C.; Bluman, G.W. Derivation of conservation laws from nonlocal symmetries of differential equations. J. Math. Phys. 1996, 37, 2361-2375. [CrossRef]

7. Anco, S.C.; Bluman, G.W. Direct construction of conservation laws from field equation. Phys. Rev. Lett. 1997, 78, 869-2873. [CrossRef]

8. Anco, S.C.; Bluman, G.W. Direct construction method for conservation laws of partial differential equations. Part I: Examples of conservation law classifications. Eur. J. Appl. Math. 2002, 13, 545-566. [CrossRef]

9. Anco, S.C.; Bluman, G.W. Direct construction method for conservation laws of partial differential equations. Part II: General treatment. Eur. J. Appl. Math. 2002, 13, 567-585. [CrossRef]

10. Bluman, G.W.; Temuerchaolu; Anco, S.C. New conservation laws obtained directly from symmetry action on a known conservation law. J. Math. Anal. Appl. 2006, 322, 233-250. [CrossRef]

11. Ibragimov, N.H. A new conservation theorem. J. Math. Anal. Appl. 2007, 333, 311-328. [CrossRef]

12. Anco, S.C. On the incompleteness of Ibragimov's conservation law theorem and its equivalence to a standard formula using symmetries and adjoint-symmetrie. Symmetry 2017, 9, 33. [CrossRef]

13. Cheviakov, A.F.; Naz, R. A recursion formula for the construction of local conservation laws of differential equations. J. Math.Anal.Appl. 2017, 448, 198-212. [CrossRef]

14. Wolf, T. A comparison of four approaches to the calculation of conservation laws. Eur. J. Appl. Math. 2002, 13, 129-152. [CrossRef]

15. Hereman, W. Symbolic computation of conservation laws of nonlinear partial differential equations in multi-dimensions. Int. J. Quantum Chem. 2006, 106, 278-299. [CrossRef]

16. Temuerchaolu; Bluman, G.W. An algorithmic method for showing existence of non-trivial non-classical symmetries of partial differential equations without solving determining equations. J. Math. Anal. Appl. 2014, 411, 281-296. [CrossRef]

17. Cheviakov, A.F. GeM software package for computation of symmetries and conservation laws of differential equations. Comput. Phys. Commun. 2007, 176, 48-61. [CrossRef]

18. Buhe, E.; Bluman, G.W. Symmetry reductions, exact solutions, and conservation laws of the generalized Zakharov equations. J. Math. Phys. 2015, 56, 101501-101514. [CrossRef]

19. Zhang, Z.Y. On the existence of conservation law multipliers for partial differential equations. Commun. Nonlinear Sci. Numer. Simulat. 2015, 20, 338-351. [CrossRef]

20. Buhe, E.; Bluman, G.W.; Kara, A.H. Conservation laws for some systems of nonlinear PDEs via the symmetry/adjoint symmetry pair method. J. Math. Anal. Appl. 2016, 436, 94-103. [CrossRef]

21. Sarlet, W.; Cantrijn, F.; Crampin, M. Pseudo-symmetries, Noether's theorem and the adjoint equations. J. Phys. A 1987, 20, 1365-1376. [CrossRef]

22. Sarlet, W.; Prince, G.E.; Crampin, M. Adjoint symmetries for time-independent second-order equations. J. Phys. A 1990, 23, 1335-1347. [CrossRef]

23. Ma, W.X. Conservation laws of discrete evolution equations by symmetries and adjoint symmetries. Symmetry 2015, 7, 714-725. [CrossRef]

24. Ibragimov, N.H. Transformation Groups Applied to Mathematical Physics; Reidel: Boston, MA, USA, 1985.

25. Bluman, G.W; Temeuerchaolu. Conservation laws for nonlinear telegraph equations. J. Math. Anal. Appl. 2005, 310, 459-476. [CrossRef]

26. Bluman, G.W.; Temuerchaolu; Sahadevan, R. Local and nonlocal symmetries for nonlinear telegraph equations. J. Math. Phys. 2005, 46. [CrossRef] 
27. Bluman, G.W.; Temuerchaolu. Comparing symmetries and conservation laws of nonlinear telegraph equations. J. Math. Phys. 2005, 46. [CrossRef]

28. Ibragimov, N.H. Invariant Lagrangians and a new method of integration of nonlinear equations. J. Math. Anal. Appl. 2005, 304, 212-235. [CrossRef] 